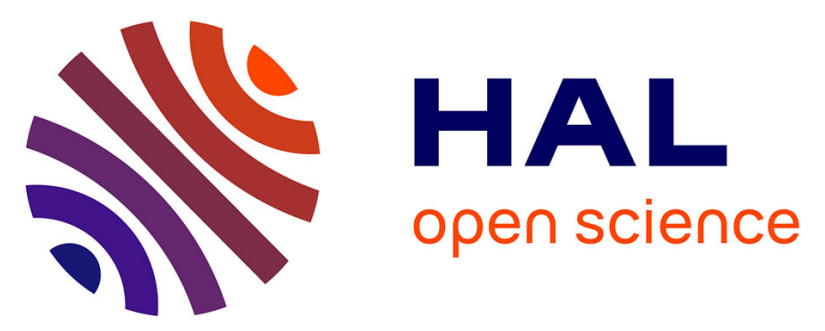

\title{
Analysis of $\mathrm{P}$ band radar signal potential to retrieve soil moisture profile
}

\author{
Mehrez Zribi, M. Sahnoun, Richard Dusséaux, Saddek Afifi, Nicolas \\ Baghdadi, A. Benhamida
}

\section{- To cite this version:}

Mehrez Zribi, M. Sahnoun, Richard Dusséaux, Saddek Afifi, Nicolas Baghdadi, et al.. Analysis of P band radar signal potential to retrieve soil moisture profile. 2nd International Conference on Advanced Technologies for Signal and Image Processing (ATSIP), Mar 2016, Monastir, Tunisia. pp.591-595, 10.1109/ATSIP.2016.7523150 . insu-01355272

\section{HAL Id: insu-01355272 \\ https://hal-insu.archives-ouvertes.fr/insu-01355272}

Submitted on 14 Jan 2022

HAL is a multi-disciplinary open access archive for the deposit and dissemination of scientific research documents, whether they are published or not. The documents may come from teaching and research institutions in France or abroad, or from public or private research centers.
L'archive ouverte pluridisciplinaire HAL, est destinée au dépôt et à la diffusion de documents scientifiques de niveau recherche, publiés ou non, émanant des établissements d'enseignement et de recherche français ou étrangers, des laboratoires publics ou privés.

\section{(ㅇ)(1) $\$$}

Distributed under a Creative Commons Attribution - NonCommerciall 4.0 International 


\title{
Analysis of $\mathrm{P}$ band radar signal potential to retrieve soil moisture profile
}

\author{
M. Zribi ${ }^{1}$, M. Sahnoun ${ }^{12}$, R. Dusséaux ${ }^{3}$, S. Afifi ${ }^{4}$ N. Baghdadi ${ }^{5}$, A. Ben Hamida ${ }^{2}$ \\ ${ }^{l}$ CESBIO (CNRS/UPS/IRD/CNES), 18 av. Edouard Belin, bpi 2801, 31401 Toulouse cedex9, France. \\ ${ }^{2}$ ENIS/Université de Sfax, Route Soukra Km 3.5, Sfax, Tunisia. \\ ${ }^{3}$ LATMOS (CNRS/IPSL), Université de Versailles Saint-Quentin en Yvelines/ Université Paris-Sacaly, 11 boulevard d'Alembert, \\ 78280 Guyancourt, France. \\ ${ }^{4}$ LAPLASO, Badji Mokhtar-Annaba University, P.O. Box 12, 23000 Annaba, Algeria \\ ${ }^{5}$ IRSTEA, UMR TETIS, 500 rue François Breton, 34093 Montpellier cedex 5, France.
}

\begin{abstract}
In this paper, we propose an analysis of $\mathbf{P}$ band radar signal potential to retrieve soil moisture root profile. Our analysis is based on two electromagnetic models, the small perturbation method and the small slope approximation. There models consider electromagnetic scattering from threedimensional layered structures with an arbitrary number of rough surfaces. Simulations are proposed for different types of moisture profiles, for different hydrological conditions.
\end{abstract}

Keywords-component; Moisture profile, rough layered surfaces, small perturbation model, Small Slope Approximation

\section{INTRODUCTION}

Soil moisture is an essential parameter for the description of different phenomena linked to surface hydrology. In the last years, different soil-vegetation-atmosphere Transfert models have assimilated soil moisture to improve simulation of surface fluxes, and particularly evapotransipration estimation, and to understand its relationship with the vegetation cover and the soil's water content [Albergel et al., 2010].

Microwave remote sensing has demonstrated its strong potential for monitoring of soil moisture over the last twenty years [Paris et al., 2008, Zribi et al., 2011, Baghdadi et al., 2012]. Using Synthetic Aperture Radars (SARs), it is possible to estimate the soil moisture at a high spatial resolution. The existing radar data cover principally three frequency bands, L $(\sim 1.25 \mathrm{GHz}), \mathrm{C}(\sim 5.3 \mathrm{GHz})$ and $\mathrm{X}(\sim 9.25 \mathrm{GHz})$. Numerous theoretical and experimental studies have shown the potential of these data to map surface soil moisture. For these frequencies, penetration depth is often considered between 5 and $2 \mathrm{~cm}$. (Morvan et al., 2008, Zribi et al., 2014) have also analyzed vertical heterogeneities of moisture in this limited profile as a function of frequency. In spite the high contribution of surface moisture, assimilated in models describing soilvegetation-atmosphere interface processes, the knowledge of moisture root profile is certainly more important for all these models. Using only surface moisture products, we consider generally a coupling process model to deduce the root moisture profile. With the arriving of BIOMASS P-band radar mission, approximately in 2023, the contribution of radar data to estimate moisture root profiles could be improved. In fact, penetration depth could rich more than $1 \mathrm{~m}$ for dry soils. In this context, the objective of this paper is to discuss potential of these future data to estimate moisture profile, through simulations considering two three-dimensional layered backscattering models.

The second section presents the two considered models for backscattering simulations. The third section is dedicated to discussion of simulations for different hydrological conditions and moisture profiles.

\section{PRINCIPES OF SPM AND SSA MODELS}

\section{A. Statistical description of surfaces}

The stratified medium under consideration is an arbitrary plane-layered medium with one rough interface on the top. Two consecutive interfaces $(i$ and $i+1)$ are separated by a layer, spatially homogeneous and isotropic, with thickness $d_{i}$. We consider $N-1$ interfaces and $N$ layers. The two-dimensional interface air/soil is characterized by a Gaussian height distribution with zero mean value and an isotropic and exponential autocorrelation function $R(x, y)$ :

$$
R(x, y)=s^{2} \exp \left(-\frac{\sqrt{x^{2}+y^{2}}}{l}\right)
$$

$s$ is the rms height of the interface air/soil and $l$, the correlation length, respectively. The spectrum $\hat{R}(\alpha, \beta)$ corresponds to the Fourier Transform (FT) of $R(x, y)$ with:

$$
\hat{R}(\alpha, \beta)=\frac{4 \pi s^{2} l^{2}}{{\sqrt{1+\left(\alpha^{2}+\beta^{2}\right) l^{2}}}^{3}}
$$

\section{B. Small perturbation method and small slope approximation}

The stratified medium is illuminated with a monochromatic plane wave. The incident wave vector $\boldsymbol{k}_{\boldsymbol{0}}$ is defined by its zenith angle $\theta_{0}$ and its azimuth angle $\phi_{0}$. The components of $\boldsymbol{k}_{\boldsymbol{\theta}}$ in a Cartesian coordinate system are $\left(\alpha_{0}, \beta_{0},-\gamma_{0}\right)$ :

$$
\alpha_{0}=k \sin \theta_{0} \cos \phi_{0}, \beta_{0}=k \sin \theta_{0} \sin \phi_{0}, \gamma_{0}=k \cos \theta_{0}
$$

$k$ is the wave number in the air. The incident electric and magnetic field vectors, $\boldsymbol{E}_{\boldsymbol{0}}$ and $\boldsymbol{H}_{\boldsymbol{0}}$, are given by:

$$
\begin{aligned}
& \mathbf{E}_{0}=\left(A_{0(h)} \mathbf{h}_{0}+A_{0(v)} \mathbf{v}_{0}\right) \exp \left(-j \mathbf{k}_{0} \cdot \mathbf{r}\right) \\
& \mathbf{H}_{0}=\frac{1}{Z}\left(-A_{0(h)} \mathbf{v}_{0}+A_{0(v)} \mathbf{h}_{0}\right) \exp \left(-j \mathbf{k}_{0} . \mathbf{r}\right)
\end{aligned}
$$


$\boldsymbol{r}(x, y, z)$ is the position vector in the Cartesian coordinate system. $\boldsymbol{h}_{\boldsymbol{0}}$ and $\boldsymbol{v}_{\boldsymbol{0}}$ are the polarization vectors [1]. $\boldsymbol{Z}$ is the air impedance. For an incident wave with a horizontal polarization, $\boldsymbol{A}_{\boldsymbol{O}(\boldsymbol{h})}=1$ and $\boldsymbol{A}_{\boldsymbol{O}(\boldsymbol{v})}=1$. For the vertical polarization, $\boldsymbol{A}_{\boldsymbol{O}(\boldsymbol{h})}=0$ and $\boldsymbol{A}_{\boldsymbol{O}(\boldsymbol{v})}=1$. The medium illuminated with a polarized plane wave (a) generates a scattered wave with a co-polarized component (aa) and a cross-polarized component (ba). At a distance located far from the surface with a zenith angle $\theta$ and an azimuth angle $\phi$, the coherent intensity $I_{c,(b a)}(\theta, \phi)$ is proportional to the square modulus of the statistical average of the complex scattered amplitude $A_{(a a)}(\theta, \phi)$ [1]:

$$
I_{c,(b a)}(\theta, \phi)=\lim _{L \rightarrow+\infty} \frac{\left|<A_{(b a)}(\theta, \phi)>\right|^{2} \cos ^{2} \theta}{\cos \theta_{0} \lambda^{2} L^{2}\left|A_{0(a)}\right|^{2}}
$$

$L$ defines the surface area. For the study with both analytical models, $L$ is extended to infinity. The brackets $<>$ stand for a statistical average. The statistical average of scattering intensity is proportional to the statistical average of the square modulus of the scattered amplitude:

$$
I_{(b a)}(\theta, \phi)=\lim _{L \rightarrow+\infty} \frac{<\left|A_{(b a)}(\theta, \phi)\right|^{2}>\cos ^{2} \theta}{\cos \theta_{0} \lambda^{2} L^{2}\left|A_{0(a)}\right|^{2}}
$$

The incoherent intensity $I_{f,(b a)}(\theta, \phi)$ is the difference between (7) and (6) [1]:

$$
I_{f,(b a)}(\theta, \phi)=I_{(b a)}(\theta, \phi)-I_{c,(b a)}(\theta, \phi)
$$

For numerical applications, we define the normalized radar cross section (NRCS) as follows:

$$
\sigma_{(b a)}(\theta, \phi)=4 \pi \cos \theta_{0} I_{f,(b a)}(\theta, \phi)
$$

The backscattering NRCS is $\sigma_{0}\left(\theta_{0}\right)=\sigma\left(\theta=-\theta_{0}, \phi=\phi_{0}\right)$. For a stratified medium with an arbitrary number of interfaces, the scattered amplitude $A_{(a a)}(\theta, \phi)$ can be evaluated by means of a perturbation method. In [2]-[5], the electromagnetic fields inside the different layers are represented as continuous sums of plane waves, commonly called Rayleigh expansions. The scattered amplitudes in each layer are derived from a perturbation method applied to the boundary conditions. The scattered amplitude $A_{(b a)}(\theta, \phi)$ in the air is given by:

$$
A_{(b a)}^{(S P M)}(\theta, \phi)=4 \pi^{2} A_{(a)}^{(0)}\left(\alpha_{0}, \beta_{0}\right) \delta(\zeta) \delta(\xi)+A_{(b a)}^{(1)}(\alpha, \beta)
$$

with $\zeta=\alpha-\alpha_{0} ; \zeta=\beta-\beta_{0} ; \alpha=k \sin \theta \cos \phi ; \beta=k \sin \theta \sin \phi$.

$\delta$ ( $\zeta$ ) designates the Dirac distribution. The zeroth-order amplitude $A^{(0}(a)$ is the reflecting Fresnel coefficient for a planar structure. At the zeroth order, the wave propagates in the specular direction and is not depolarized. For a plane-layered stack with one rough interface on the top, the first-order scattered amplitude $A^{(1)}$ (ba) is given by:

$$
A_{(b a)}^{(1)}=K_{1(b a)}(\alpha, \beta) \hat{a}(\zeta, \xi)
$$

$\hat{a}(\alpha, \beta)$ is the Fourier transform of the function $a(x, y)$ describing the interface air/soil. The factor $K_{1(b a)}(\alpha, \beta)$ is given by a recurrence formula [4]-[5]. In fact, in order to express the scattering amplitude in the air, the first-order perturbation method requires the determination of $N-1$ factors $K_{i(b a)}(\alpha, \beta)$ associated with $N-1$ interfaces.

Factors $K i_{\text {(ba) }}(\alpha, \beta)$ with $2 \leq \mathrm{i} \leq N-1$ do not appear in (10) because we consider a plane-layered medium with one rough interface on the top. These factors depend on the relative permittivities and the thicknesses of the different layers, and on the zenith and azimuth angles of incident and scattered waves. We derive from (6)-(11) the NRCS which depends on the spectrum of the interface air/soil.

$$
\sigma_{(b a)}^{(S P M)}(\theta, \phi)=\frac{4 \pi \cos ^{2} \theta}{\lambda^{2}}\left|K_{1(b a)}\right|^{2} \hat{R}(\zeta, \xi)
$$

The small slope approximation method at the first order gives the scattered wave amplitude in the form of an integral where the integrant depends on the kernel of the small perturbation method at the first order. Knowing the electromagnetic kernel from the small perturbation method, we can deduce the expression of the scattered amplitude within the framework of the SSA method [6]. In [2] and [3], from the kernels given by SPM, the SSA is extended at the first order for a stratified medium with an arbitrary number of interfaces. For a plane-layered medium with rough upper interface, we obtain the complex scattered amplitude as follows:

$$
\begin{aligned}
A_{(b a)}^{(S S A)}(\alpha, \beta)= & A_{0(a)} \frac{K_{1(b a)}(\alpha, \beta)}{j\left(\gamma+\gamma_{0}\right)} J(\alpha, \beta) \\
& +A_{0(a)} 4 \pi^{2} \sum_{i=2}^{N-1} \frac{K_{i(b a)}\left(\alpha_{0}, \beta_{0}\right)}{2 j \gamma_{0}} \delta(\zeta) \delta(\xi)
\end{aligned}
$$

with

$$
J=\int_{-\infty}^{+\infty} \int_{-\infty}^{+\infty} \exp (j \zeta x) \exp (j \xi y) \exp \left[j\left(\gamma_{1}+\gamma_{0}\right) a(x, y)\right] d x d y
$$

Where $\gamma=k \cos \theta$. The SSA method leads to the SPM expressions when the roughness becomes smaller than the wavelength. The solution (14) is consistent with the expression (10) if:

$$
A_{(a)}^{(0)}= \begin{cases}\sum_{i=1}^{N-1} \frac{K_{i(a a)}\left(\alpha_{0}, \beta_{0}\right)}{j 2 \gamma_{0}} & \text { if }(\mathrm{a})=(\mathrm{b}) \\ 0 & \text { if }(\mathrm{a}) \neq(\mathrm{b})\end{cases}
$$

We find from (6)-(8) and (14) the NRCS:

$$
\sigma_{(a b)}^{(S S A)}(\theta, \phi)=\frac{\gamma^{2}}{\pi\left(\gamma+\gamma_{0}\right)^{2}}\left|K_{1,(b a)}\right|^{2} \exp \left[-S^{2}\left(\gamma+\gamma_{0}\right)^{2}\right] P
$$

with

$$
P=\sum_{q=1}^{+\infty} \frac{\left(\gamma+\gamma_{0}\right)^{2 q}}{q !} F T\left[R^{q}(x, y)\right](\zeta, \xi)
$$

For an isotropic and exponential autocorrelation function, we obtain: 


$$
P=\sum_{q=1}^{+\infty} \frac{\left(\gamma+\gamma_{0}\right)^{2 q}}{q !} \frac{4 \pi s^{2 q} l_{q}^{2}}{\sqrt{1+\left(\zeta^{2}+\xi^{2}\right) l_{q}^{2}}}
$$

where $l_{q}=l / q$. For small roughness, the relations (16)-(17) derived at the second order lead to the bistatic coefficient obtained with the small perturbation method (Eq. 12). As with the first-order SPM, there is no depolarization in the incidence plane and within the framework of SSA at the first order, the cross-polarized intensities are equal to zero in the plane $\phi=\phi_{0}$.

\section{BACKSCATTERING SIMULATION RESULTS}

\section{A. Generated soil moisture profiles}

In this section, we generate different types of soil moisture profile. The objective of this first step is to consider different hydrological conditions from dry to wet conditions. The three first moisture profile types are described analytically with a simple second order polynomial function:

$$
m v(z)=a z^{2}+b z+c
$$

With $z$, the depth of soil.

Figure 1-a illustrates profiles with low moisture profile at surface level and then an increasing of soil moisture function of depth (type1). This condition is generally retrieved a long period after wet conditions, when deep depths are wettest than surface. Figure 1-b illustrates a second case of moisture profiles (type2) with a decreasing of soil moisture function of depth. These conditions could be retrieved for example just after important rainfall events. The surface level is very wet and soil moisture is lower for deep areas. Figure 1-c illustrates another type of moisture vertical profile (type3). For this one, we consider a vertical variation generally retrieved at dry season, for which the highest level of moisture is retrieved for deepest areas with a dry surface.

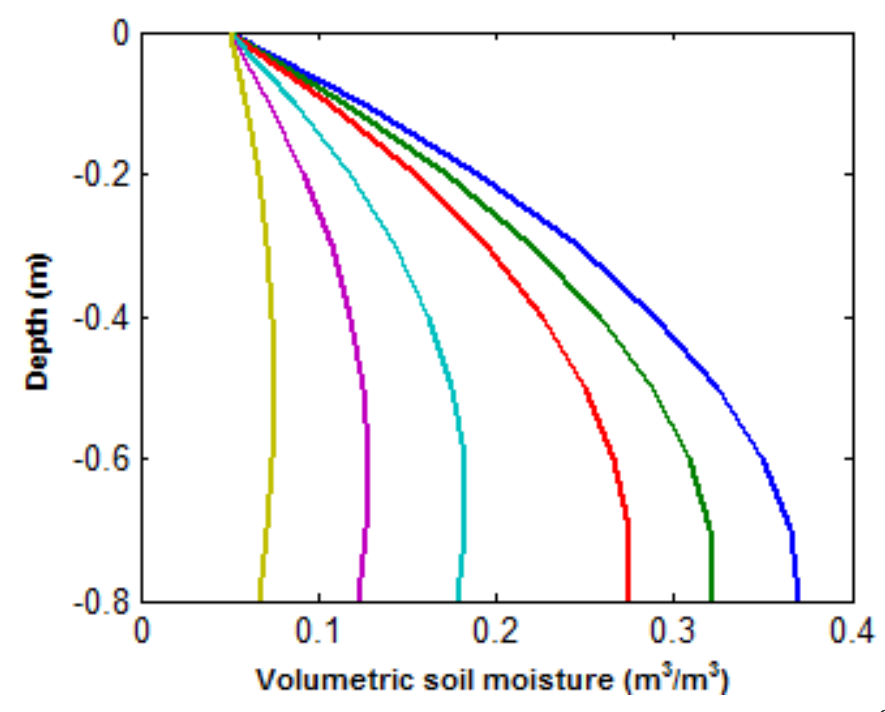

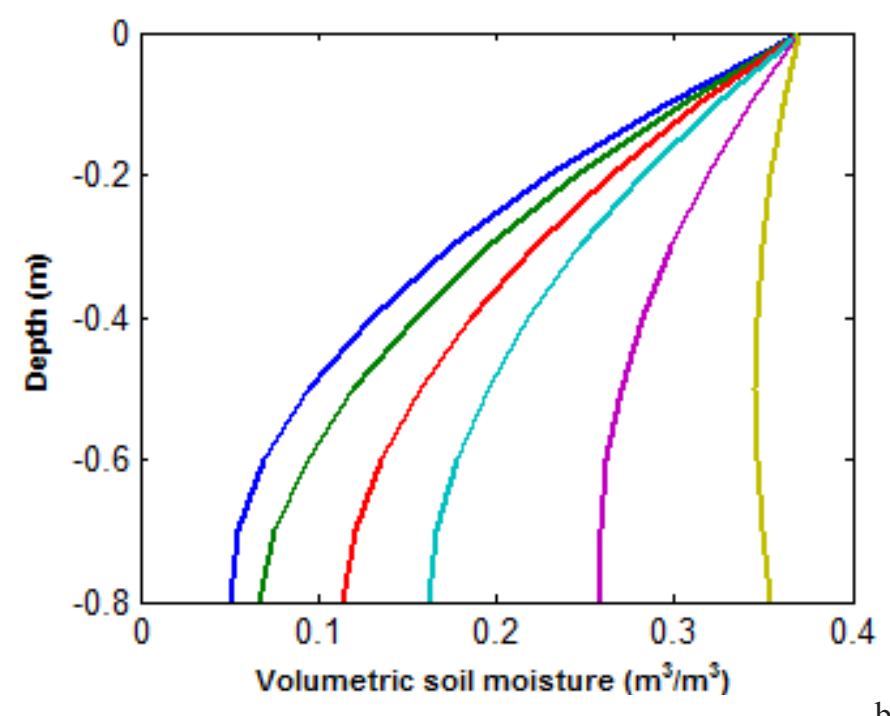

$\mathrm{b}$

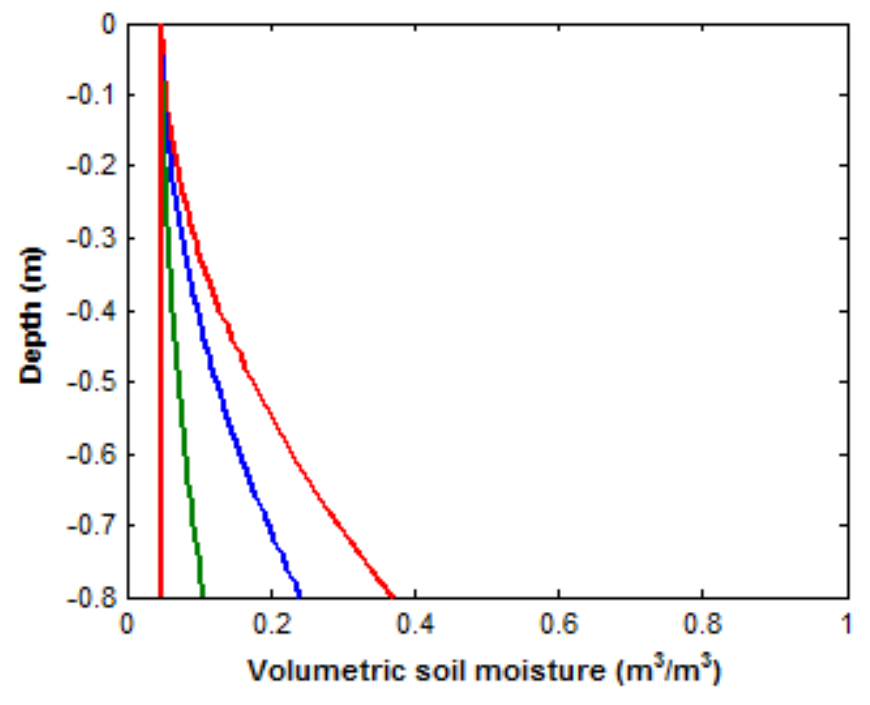

Fig. 1. Generated moisture vertical profiles,with (a) an increasing of moisture as a function of depth (type1) (b) a decreasing of moisture as a function of depth (type2), (c) an increasing of moisture function of depth (type3)

The final moisture profile type (type4) is simulated using a gaussian analytical function (figure 2). This type of profile is frequent in wet season. It can be measured some days after a wet event. A maximum of moisture is then retrieved at a certain depth with a decreasing of moisture level for highest and lowest depths. The profile could be written as :

$$
m v(z)=M v_{\max } \exp \left(-\frac{(z-z c)^{2}}{2 \sigma^{2}}\right)
$$

where $\mathrm{Mv}_{\max }$ is the maximum of moisture at zc depth and $\sigma$, the standard deviation of Gaussian function, respectively. 


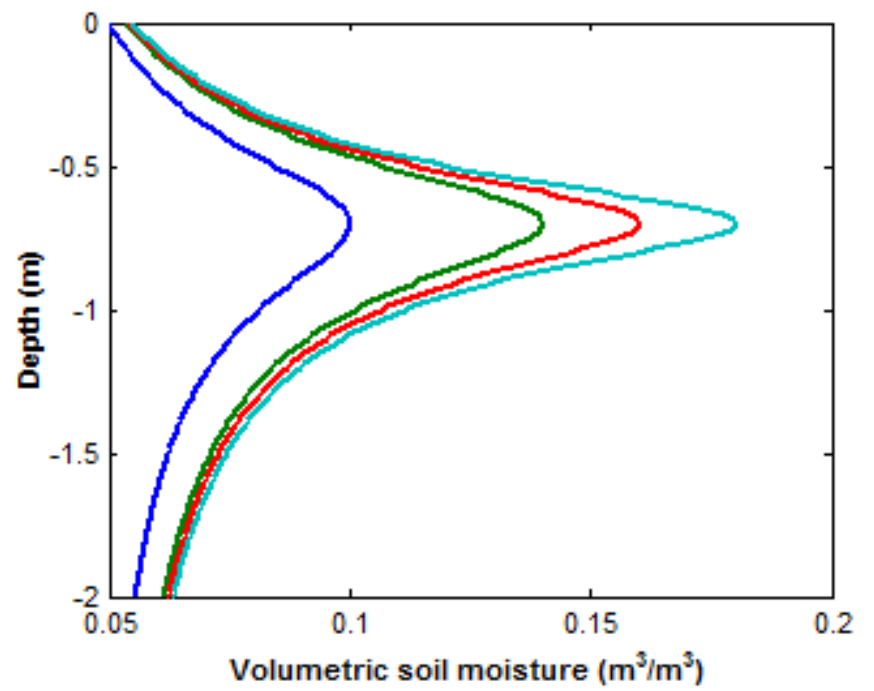

Fig. 2. Generated moisture vertical profiles with a gaussian behaviour (type4)

All these profiles are considered to analyze the potential of $\mathrm{P}$ band radar data to retrieve moisture vertical profile. Dielectric constant, directly linked to soil moisture, is the input of backscattering models. In this case, we consider the empirical models developed by (Peplinski et al., 1995a and Peplinski et al., 1995b), for frequency interval (0.3-1 GHz).

\section{B. Discussion of model convergence}

The first step, before the analysis of radar signal behavior function of soil moisture profile, is to retrieve the minimum number of layers for a convergence of backscattered signal computations.

For the four types of profiles, simulations are proposed as a function of the number of layers $(\mathrm{N})$, for a constant profile depth equal to $0.8 \mathrm{~m}$. As illustrated by figure 3, for all profiles, we retrieve first a variation of backscattering signal with the increasing of $\mathrm{N}$, and then a convergence of estimated signals. With limited number of layers, probably the effects of driest depths are less considered and then signal level is higher. This convergence is retrieved approximately for $\mathrm{N}$ between 15 and 20. Based on these results, we consider for all simulations a number of layers equal to 20 .

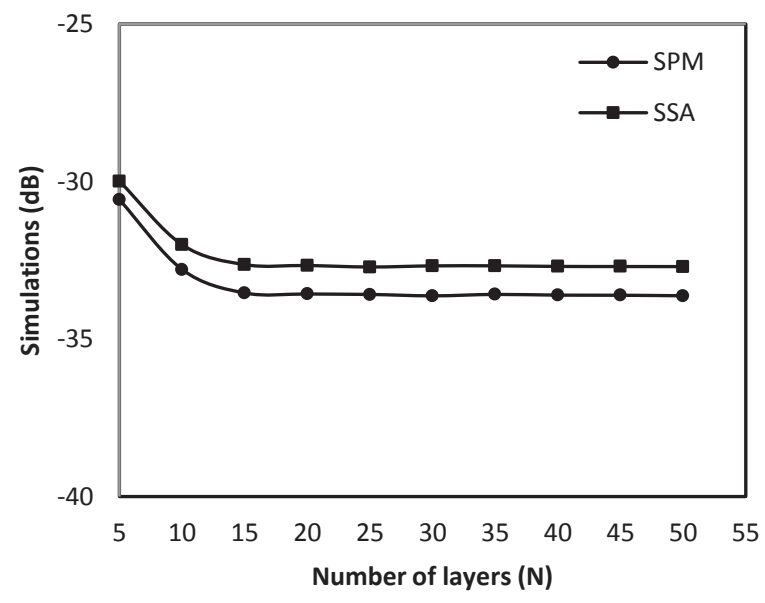

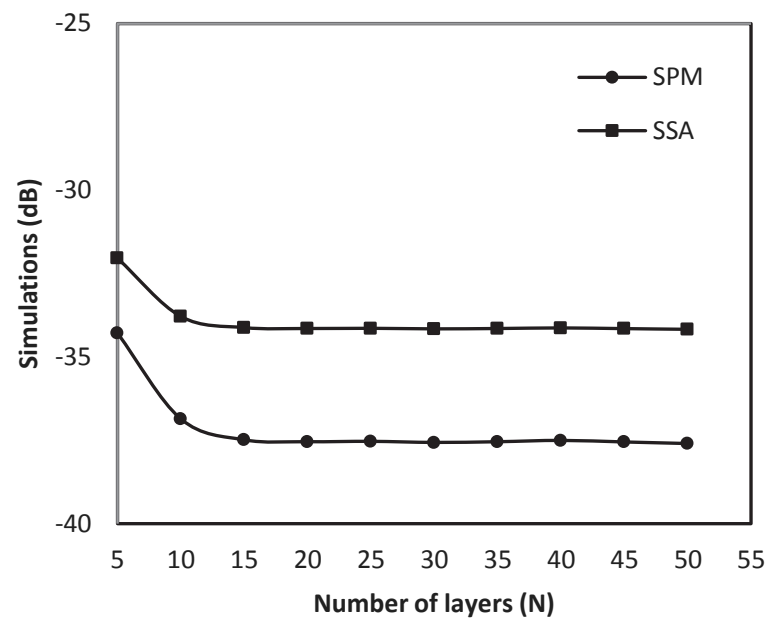

$\mathrm{b}$

Fig. 3. Simulations of backscattering function of number of layers $\mathrm{N}$ (a) $\mathrm{HH}$ polarization, (b) VV polarization

\section{Backscattering simulations with SPM and SSA models}

Backscattering simulations with SPM and SSA models are realized for all moisture profiles. Figures 4 illustrate results of simulations for all proposed moisture conditions. For profiles with type 1 (figure 4-a), with a dry surface and an increasing of moisture level function of depth, we observe a maximum of difference, between the two extreme profiles, approximately equal to $2.5 \mathrm{~dB}$ for all incidence angles. When surface is wet (the case of profiles with type2), the difference between all profiles is limited (about $1 \mathrm{~dB}$ ), figure 4-b. This last result is linked to the limited wave penetration depth in the case of wet medium. The third illustration (type3, figure 4-c) shows a difference between the two extreme profiles equal to $0.6 \mathrm{~dB}$. For this case, difference is lower than those of type 1 profiles, because moisture differences are limited for the first $40 \mathrm{~cm}$ depth.

The fourth illustration concerns the Gaussian type of profiles, with a maximum of soil moisture in a certain depth. For this case, we retrieve a medium difference between simulations equal to $1.2 \mathrm{~dB}$.

For all results, we observe some discrepancies between the two tested models SPM and SSA, probably because of differences in validity domain and physical approximations introduced for each model.

All these results confirm the potential of $\mathrm{P}$ band to have details about moisture vertical profile in the case of dry conditions at surface, allowing important penetration of waves. This case is particularly important, because of difficulty to estimate moisture profile in dry or intermediate meteorological conditions.

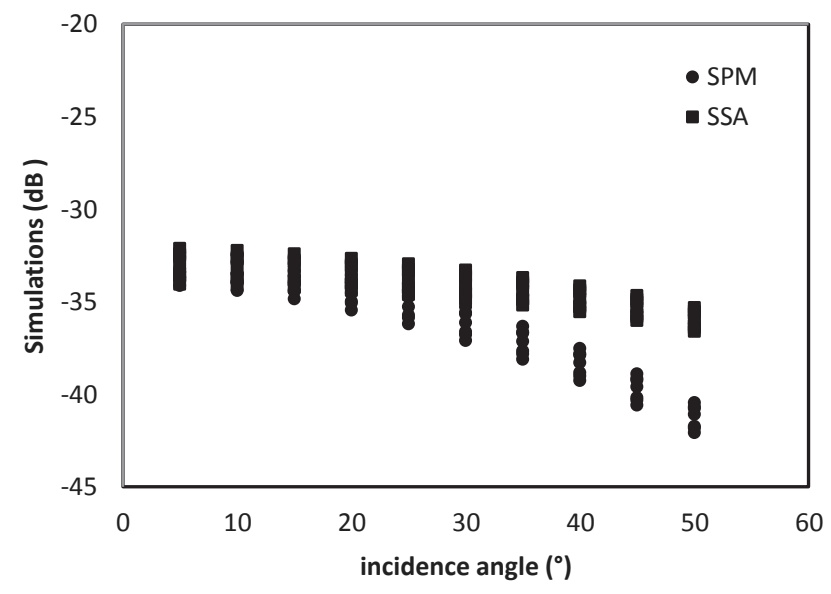




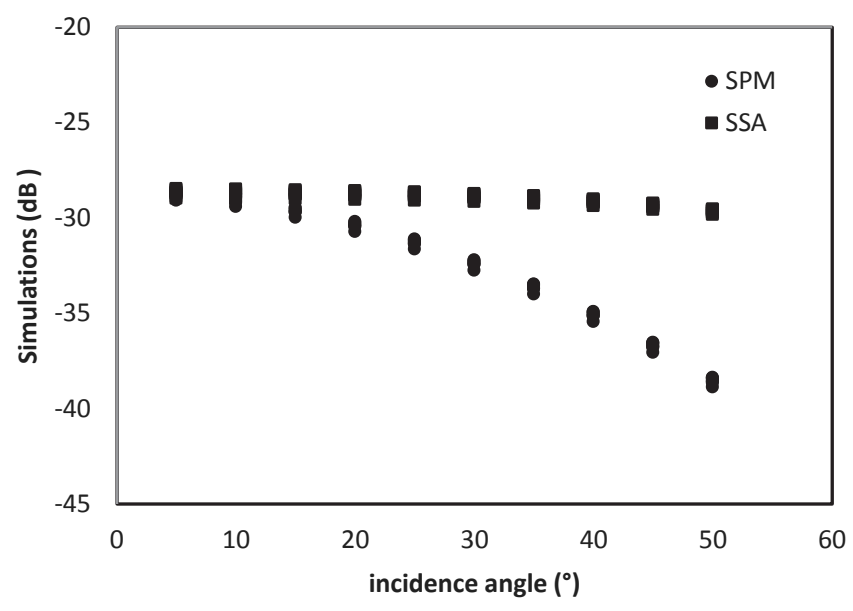

b

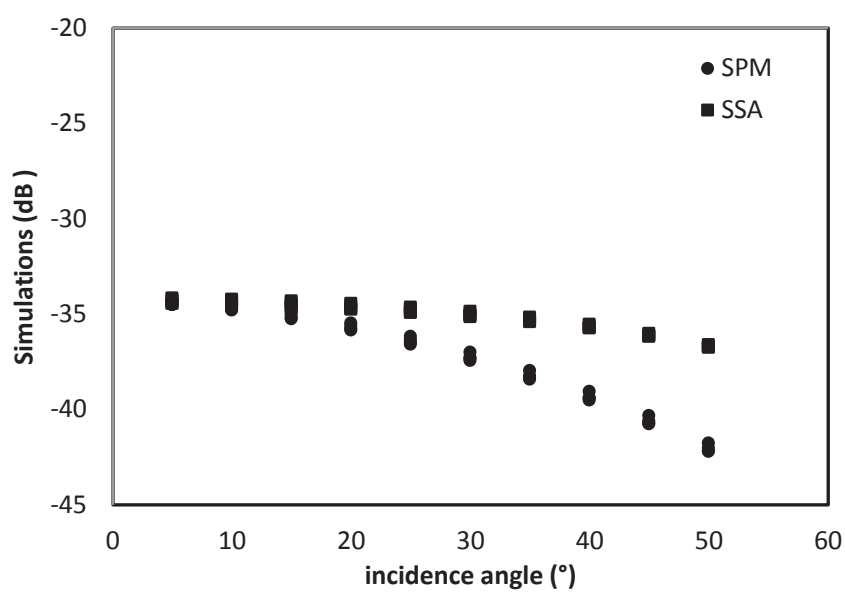

$\mathrm{c}$

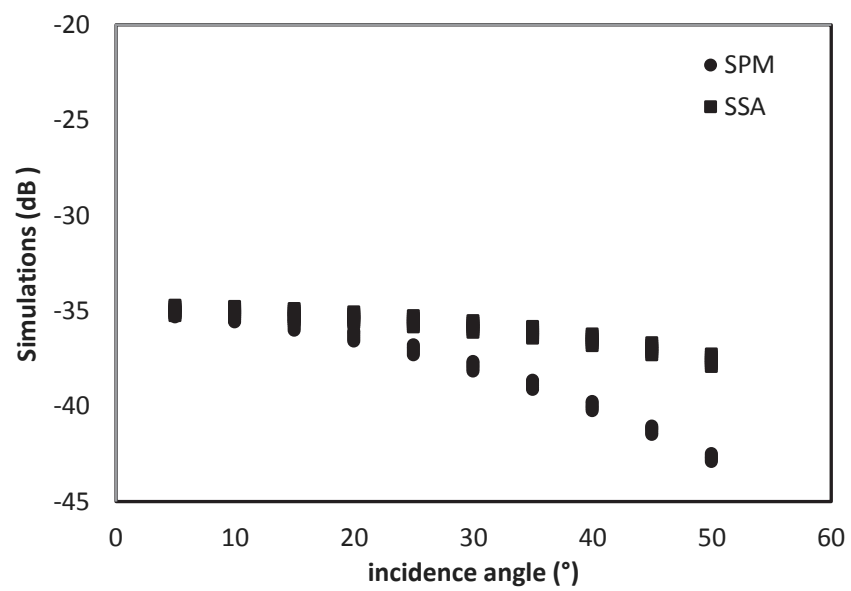

Fig. 4. Simulations of backscattering coefficient function of incidence angles for the two studied models (SPM and SSA), case of HH polarization a) profiles type1, b) profiles type 2, c) profiles type 3, d) profiles type 4

\section{CONCLUSIONS}

This paper has considered backscattering simulations for different moisture conditions to analyze potential of P-band radar data to retrieve moisture vertical profiles. Simulations are based on two multi-layer models SPM and SSA. Discussions have shown the high potential of $\mathrm{P}$ band to retrieve some types of moisture vertical profiles, those for which soil surface is dry. In this case, discrepancy between extreme profiles could reach $2.5 \mathrm{~dB}$. These results still preliminary and need to be validated by other studies considering experimental campaigns with $\mathrm{P}$ band real data and different ground measurement conditions.

\section{ACKNOWLEDGMENT}

This study was funded by the TOSCA/CNES ASCAS project. The authors thank the "Programme National de Télédétection Spatiale" that has supported the research of this paper (AO2015- 880238).

\section{REFERENCES}

[1] Albergel, C., Calvet, J.-C., de Rosnay, P., Balsamo, G., Wagner, W., Hasenauer, S., Naeimi, V., Martin, E., Bazile, E., Bouyssel, F., and Mahfouf, J.-F.: Cross-evaluation of modelled and remotely sense surface soil moisture with in situ data in southwestern France, Hydrol. Earth Syst. Sci., 14, 2177-2191, doi:10.5194/hess-14-2177-2010, 2010.

[2] Paris Anguela, T., Zribi, M., Habets, F., Hasenauer, S., and Loumagne, C., 2008, "Analysis of surface and root soil moisture dynamics with ERS scatterometer and the hydrometeorological model SAFRAN-ISBAMODCOU at Grand Morin watershed (France)", HESS (Hydrology and Earth System Sciences ), Special Issue on Remote sensing in hydrological sciences, 5, 1903-1926.

[3] Zribi, M., Pardé, M., Boutin, J., Fanise, P., Hauser, D., Dechambre, M., Kerr, Y., Leduc-Leballeur, M., Reverdin, G., Skou, N., SøbjærG, S., Albergel, C., J. C. Calvet, J. P. Wigneron, E. Lopez- Baeza, A. Ruis and J. Tenerelli, 2011, "CAROLS: A New Airborne L-Band Radiometer for Ocean Surface and Land Observations", Sensors, 11(1), 719-742; doi:10.3390/s110100719.

[4] Baghdadi N., Abou Chaaya J., and Zribi M., 2011. Semi-empirical calibration of the Integral quation Model for SAR data in C-band and cross polarization using radar images and field measurements. IEEE Geoscience and Remote Sensing Letters, vol. 8, no.1, pp.14-18.

[5] Le Morvan, A., Zribi, M., Baghdadi, N., and Chanzy, A., 2008, "Soil moisture profile effect on radar signal measurement, Journal of Sensors", Sensors, 8, 256-270.

[6] Zribi, M., Gorrab, A., Baghdadi, N., Lili-Chabaane, Z., Mougenot, B., Influence of radar frequency on the relationship between bare surface soil moisture vertical profile and radar backscatter, IEEE Geoscience and Remote Sensing Letters, 11(4) 848 - 852 10.1109/LGRS.2013.2279893.

[7] L. Tsang and J.A. Kong, Scattering of Electromagnetic Waves Advanced Topics. New York: Wiley-Interscience, 2001

[8] S. Afifi and R. Dusséaux, "On the co-polarized phase difference of rough layered surfaces: Formulas derived from the small perturbation method," IEEE Trans. Antennas Propagat., vol. 59, no. 7, pp 2607-2618, July 2011.

[9] S. Afifi and R. Dusséaux, "On the co-polarized scattered intensity ratio of rough layered surfaces: The probability law derived from the small perturbation method," IEEE Trans. Antennas Propagat., vol. 60, no. 4, pp 2133-2138, April 2012.

[10] A.Berrouk, R.Dusséaux, S.Afifi, "Electromagnetic wave scattering from rough layered interfaces: analysis with the small perturbation method and the small slope approximation," Progress In Electromagnetics Research B, Vol. 57, pp. 177-190, 2014.

[11] S. Afifi, R. Dusséaux, A. Berrouk, "Electromagnetic wave scattering from 3D layered structures with randomly rough interfaces: analysis with the small perturbation method and the small slope approximation," IEEE Antennas and Propagation, vol. 62, no. 10, pp. 5200-5208, 2014.

[12] G. Voronovich, Wave Scattering from Rough Surfaces, Springer, Berlin, 1994.

[13] Neil R. Peplinski, Fawwaz T. Ulaby, and Myron C. Dobson, Dielectric Properties of Soils in the $0.3-1.3-\mathrm{GHz}$ Range, IEEE Trans. Geosci. Remote Sens., Vol. 33, No. 3, May 1995.

[14] Neil R. Peplinski, Fawwaz T. Ulaby, and Myron C. DobsonCorrections to "Dielectric Properties of Soils in the 0.3-1.3-GHz Range", IEEE Trans. Geosci. Remote Sens., Vol. 33, No. 6, Nov. 1995. 